\title{
ALGEBRAIC SHIFTING OF FINITE GRAPHS
}

\author{
SATOSHI MURAI
}

\begin{abstract}
Exterior algebraic shifting and symmetric algebraic shifting of bipartite graphs and chordal graphs are studied in the present paper.

keywords: Algebraic shifting, chordal graph.
\end{abstract}

\section{INTRODUCTION}

Let $G=([n], E(G))$ be a graph on the vertex set $[n]=\{1,2, \ldots, n\}$ with the edge set $E(G)$. A graph $G$ is called shifted if for any edge $\{i, j\} \in E(G)$ and for any $i^{\prime} \leq i$ and $j^{\prime} \leq j$, one has $\left\{i^{\prime}, j^{\prime}\right\} \in E(G)$. Shifting operation of graphs is an operation which associate a graph $G$ with a shifted graph $\Delta(G)$. Shifting operation is first considered by Erdös, Ko, and Rado [4]. Their shifting operation is called a combinatorial shifting $\Delta^{c}(G)$. Algebraic shifting is a shifting operation which was introduced by Kalai. Main variants of algebraic shifting are exterior shifting $\Delta^{e}(G)$ and symmetric shifting $\Delta^{s}(G)$. These definitions are given in $\S 1$. We also introduce ring theoretical definitions using generic initial ideal in $\S 3$. Even though, shifting operations are defined for simplicial complex, we only consider the special case of graphs in this paper.

Algebraic shifting of graphs is first studied by G. Kalai [6]. His main arguments are relations between algebraic shifting and connectivity together with saturated chain of graphs. We are interested in differences between exterior shifting and symmetric shifting. Our main problem is when $\Delta^{e}(G)$ and $\Delta^{s}(G)$ are equal and when they are different? A complete bipartite graph $K_{3,3}$ of size 3 is one example of a graph which satisfies $\Delta^{e}\left(K_{3,3}\right) \neq \Delta^{s}\left(K_{3,3}\right)$ (This was found by Kalai). Also, we can easily show that $\Delta^{e}(G)=\Delta^{s}(G)$ if $G$ is tree. In this paper, to extend these two examples, we will study algebraic shifting of bipartite graphs and chordal graphs.

First, we consider bipartite graphs in $\S 2$. Our result determines the form of $\Delta^{s}(G)$ when $G$ is a complete bipartite graph. Kalai determined $\Delta^{e}(G)$ for complete bipartite graphs in [6]. Kalai's result and our result implies the difference between $\Delta^{e}(G)$ and $\Delta^{s}(G)$. In fact, if $G$ is a complete bipartite graph which contains $K_{3,3}$, then we have $\Delta^{c}(G)=\Delta^{e}(G) \neq \Delta^{s}(G)$, where $\Delta^{c}(G)$ is unique in this case. (Theorem 2.5 and Lemma 4.7). Also, we see $\Delta^{e}(G) \neq \Delta^{s}(G)$ if $G$ is bipartite graph and has a lot of edges (Corollary 2.6).

Second, we consider chordal graphs. We call $G$ chordal if every cycle in $G$ has a chord. For a chordal graph $G$, we can easily show $\Delta^{e}(G)=\Delta^{s}(G)$. This fact will be given in $\S 3$. 
On the other hand, we consider computations of algebraic shifting of chordal graph in $\S 4$. No effective method to compute exterior shifting and symmetric shifting is known. In fact, it is really hard to compute exterior shifting and symmetric shifting. On the other hand, combinatorial shifting is easy to compute. However, combinatorial shifting does not have a good behavior. For example, it is not even uniquely determined. We are interested in when $\Delta^{c}(G)$ and $\Delta^{e}(G)$ are equal. Generally, we can not always take $\Delta^{c}(\sigma)$ with $\Delta^{c}(\sigma)=\Delta^{e}(\sigma)$ for a simplicial complex $\sigma$ (this example is introduced by Kalai $[7, \S 6.2]$ and by us $[11, \S 4]$ ). But we do not have an example of graph $G$ which satisfies $\Delta^{c}(G) \neq \Delta^{e}(G)$ for all $\Delta^{c}(G)$. We give an algorithm to compute $\Delta^{c}(G)$ with $\Delta^{c}(G)=\Delta^{e}(G)=\Delta^{s}(G)$, when $G$ is chordal (Theorem 4.8). Also, we determine a chordal graph $G$ whose combinatorial shifting is unique (Proposition 4.4).

\section{ALGEBRAIC SHIFTING}

Let $[n]=\{1,2, \ldots, n\},\left(\begin{array}{c}{[n]} \\ 2\end{array}\right)=\{\{i, j\} \subset[n] \mid i \neq j\}, G=([n], E(G))$ the finite graph on $[n]$ with the edge set $E(G) \subset\left(\begin{array}{c}{[n]} \\ 2\end{array}\right)$.

We define $m_{\leq k}(G)=|\{\{i, j\} \in E(G) \mid \min \{i, j\} \leq k\}|$ and $m_{k}(G)=\mid\{\{i, j\} \in$ $E(G) \mid \min \{i, j\}=k\} \mid$, where $|V|$ means the number of elements of finite set $V$. If $G$ is shifted, then $m_{\leq k}(G)$ determine the shifted graph $G$.

Definition 1.1. Let $K$ be an infinite field with $\operatorname{char}(K)=0, V$ a $n$-dimensional $K$-vector space with basis $e_{1}, e_{2}, \ldots, e_{n}$. Let $g=\left(x_{i j}\right) \in G L_{n}(K)$.

(i) (exterior shifting) For $\{i, j\} \in\left(\begin{array}{c}{[n]} \\ 2\end{array}\right)$ we define a map $\varphi_{g, k}^{n}:\left(\begin{array}{c}{[n]} \\ 2\end{array}\right) \rightarrow \bigoplus^{k} V$ by

$$
\varphi_{g, k}^{n}(\{i, j\})=\bigoplus_{l=1}^{k}\left(x_{l j} e_{i}-x_{l i} e_{j}\right) .
$$

Set $\varphi_{g, k}^{n}(E(G))=\operatorname{span}\left\{\varphi_{g, k}^{n}(\{i, j\}) \mid\{i, j\} \in E(G)\right\}$. A graph $\Delta^{e}(G)=\left([n], \Delta^{e}(E(G))\right)$ is defined to be a shifted graph which satisfies

$$
m_{\leq k}\left(\Delta^{e}(G)\right)=\max _{g \in G L_{n}(K)}\left\{\operatorname{dim}_{K} \varphi_{g, k}^{n}(E(G))\right\} .
$$

(ii) (symmetric shifting) We define another map $\rho_{g, k}^{n}:\left(\begin{array}{c}{[n]} \\ 2\end{array}\right) \rightarrow \bigoplus^{k} V$ by

$$
\rho_{g, k}^{n}(\{i, j\})=\bigoplus_{l=1}^{k}\left(x_{l i}-x_{l j}\right)\left(e_{i}-e_{j}\right) .
$$

By the same way, a graph $\Delta^{s}(G)=\left([n], \Delta^{s}(E(G))\right)$ is defined to be a shifted graph which satisfies $m_{\leq k}\left(\Delta^{e}(G)\right)=\max _{g \in G L_{n}(K)}\left\{\operatorname{dim}_{K} \rho_{g, k}^{n}(E(G))\right\}$. About a selection of $g \in G L_{n}(K)$ we note the following property.

Lemma 1.2. There is a non empty Zarituki open set $U \subset G L_{n}(K)$ such that for every $g \in U$, for every graph $G$ on $[n]$ and for every $k \in[n]$

$$
\begin{aligned}
m_{\leq k}\left(\Delta^{e}(G)\right) & =\operatorname{dim}_{K} \varphi_{g, k}^{n}(E(G)) \\
\text { and } m_{\leq k}\left(\Delta^{s}(G)\right) & =\operatorname{dim}_{K} \rho_{g, k}^{n}(E(G)) .
\end{aligned}
$$


Proof. For any finite dimensional $K$-vector space $V \subset K^{k n}$, we can make a $|V| \times$ $n k$ matrix $X(V)$ whose lows are coincide with basis of $V$. On the other hand, $\operatorname{dim} V=\operatorname{rank} X(V)$ and $\operatorname{rank} X(V)$ is equal to the largest size of nonzero minor of $X(V)$. We take a graph $G$. Then minors of $X\left(\varphi_{g, k}^{n}(E(G))\right)$ can be seen as polynomials of $K\left[x_{i j}\right]_{1 \leq i, j \leq n}$. Thus for $k=1,2, \ldots, n$ we can take a Zarituki open set $U_{G, k} \subset G L_{n}(K)$ which satisfies $m_{\leq k}\left(\Delta^{e}(G)\right)=\operatorname{dim}_{K} \varphi_{g, k}^{n}(E(G))$ for all $g \in U_{G, k}$. Let $U_{G}=\bigcap_{k \in[n]} U_{G, k}$ and $U=\bigcap_{G \in \mathcal{G}(n)} U_{G}$, where $\mathcal{G}(n)$ denote the set of graphs on $[n]$. Since Zarituki open sets $U_{G, k}$ are dense, $U$ satisfies the condition of this lemma. Similarly, we can prove in the case of $\Delta^{s}(G)$.

Lemma $1.3([6, \S 3])$. Let $G$ be a finite graph on $[n]$ and $g \in G L_{n}(K)$. Then

$$
\begin{aligned}
\operatorname{dim}_{K} \varphi_{g, k}^{n}(E(G)) & \leq\left(\begin{array}{c}
n \\
2
\end{array}\right)-\left(\begin{array}{c}
n-k \\
2
\end{array}\right) \\
\text { and } \operatorname{dim}_{K} \rho_{g, k}^{n}(E(G)) & \leq\left(\begin{array}{c}
n \\
2
\end{array}\right)-\left(\begin{array}{c}
n-k \\
2
\end{array}\right) .
\end{aligned}
$$

These two lemmas guarantee $\Delta^{e}(G)$ and $\Delta^{s}(G)$ are well defined. Especially, Lemma 1.2 says there exists $g \in G L_{n}(K)$ such that $g$ makes $\Delta^{e}(G)$ and $\Delta^{s}(G)$ for every graph $G$. Shifted graph $\Delta^{e}(G)$ is called an exterior shifted graph of $G$ and $\Delta^{s}(G)$ is called a symmetric shifted graph of $G$. This definition is introduced in [6]. We will also introduce ring theoretical definition in $\S 3$.

Another definition of symmetric shifting may be useful ( similar definition can be found in [7]). For $i, j \in[n]$, we define

$$
\phi_{g, k}^{n}(\{i, j\})=\bigoplus_{l=1}^{k}\left(x_{l j} e_{i}+x_{l i} e_{j}\right) .
$$

Then $\Delta^{s}(G)$ is a shifted graph with

$$
m_{\leq k-1}\left(\Delta^{e}(G)\right)=\max _{g \in G L_{n}(K)}\left\{\operatorname{dim}_{K}\left\{\operatorname{span}\left\{\phi_{g, k}^{n}(\{i, j\}) \mid\{i, j\} \in E(G) \text { or } i=j\right\}\right\}\right\}-n \text {. }
$$

By an easy calculation we can see two types of definition of $\Delta^{s}(G)$ are equivalent.

\section{Algebraic Shifting of Bipartite graphS}

In this section we consider bipartite graphs. Algebraic shifting of bipartite graphs is interesting because for many of bipartite graphs $G$ we have $\Delta^{e}(G) \neq \Delta^{s}(G)$. Firstly, we introduce an exterior shifting of complete bipartite graph which was proved by Kalai [6].

Lemma 2.1 ([6, Theorem 6.1]). Let $G=K_{A, B}$ be the complete bipartite graph with $A \neq \emptyset, B \neq \emptyset$ and $|A|+|B|=n$. Then

$$
m_{\leq k}\left(\Delta^{e}(G)\right)= \begin{cases}k n-k^{2}, & \text { if } k \leq \min \{|A|,|B|\} \\ |A||B|, & \text { if } k>\min \{|A|,|B|\} .\end{cases}
$$

About a relation of symmetric shifting and exterior shifting of bipartite graph, we have a following lemma. 
Lemma 2.2. Let $G$ be a bipartite graph. Then we have

(1) $m_{\leq k+1}\left(\Delta^{e}(G)\right) \geq m_{\leq k}\left(\Delta^{s}(G)\right) \geq m_{\leq k+1}\left(\Delta^{e}(G)\right)-n+\min \left\{\left(\begin{array}{c}k+2 \\ 2\end{array}\right), n\right\}$

Proof. Assume $G \subset K_{A, B}$. Let $\left\{a_{1}, b_{1}\right\}, \ldots,\left\{a_{r}, b_{r}\right\} \in E(G)$ with $a_{i} \in A$ and $b_{i} \in B$. We take a $g \in G L_{n}(K)$ which satisfies Lemma 1.2. If $\varphi_{g, k}^{n}\left(\left\{a_{1}, b_{1}\right\}\right), \ldots, \varphi_{g, k}^{n}\left(\left\{a_{r}, b_{r}\right\}\right)$ are linearly dependent, then there exist $\alpha_{i} \in K$ for each $1 \leq i \leq r$ such that

$$
\sum_{i=1}^{r} \alpha_{i} \varphi_{g, k}^{n}\left(\left\{a_{i}, b_{i}\right\}\right)=\sum_{i=1}^{r} \alpha_{i} \bigoplus_{l=1}^{k}\left(x_{l b_{i}} e_{a_{i}}\right)-\sum_{i=1}^{r} \alpha_{i} \bigoplus_{l=1}^{k}\left(x_{l a_{i}} e_{b_{i}}\right)=0 .
$$

Thus we have

$$
\sum_{i=1}^{r} \alpha_{i} \bigoplus_{l=1}^{k}\left(x_{l b_{i}} e_{a_{i}}\right)=0 \text { and } \sum_{i=1}^{r} \alpha_{i} \bigoplus_{l=1}^{k}\left(x_{l a_{i}} e_{b_{i}}\right)=0
$$

By the definition of $\phi_{g, k}^{n}$, we have

$$
\sum_{i=1}^{r} \phi_{g, k}^{n}\left(\left\{a_{i}, b_{i}\right\}\right)=\sum_{i=1}^{r} \alpha_{i} \bigoplus_{l=1}^{k}\left(x_{l b_{i}} e_{a_{i}}\right)+\sum_{i=1}^{r} \alpha_{i} \bigoplus_{l=1}^{k}\left(x_{l a_{i}} e_{b_{i}}\right)=0 .
$$

Since $G$ is bipartite, (2),(3) and (4) are equivalent. Hence we have

$$
\operatorname{dim}_{K} \varphi_{g, k}^{n}(E(G))=\operatorname{dim}_{K} \phi_{g, k}^{n}(E(G)) .
$$

But, definition of $\Delta^{s}(G)$ says

$$
m_{\leq k}\left(\Delta^{s}(G)\right)=\operatorname{dim}_{K}\left\{\phi_{g, k+1}^{n}(E(G)) \cup \operatorname{span}\left\{\phi_{g, k+1}^{n}(\{1,1\}), \ldots, \phi_{g, k+1}^{n}(\{n, n\})\right\}\right\}-n
$$

Thus we have the first inequality of (1)

$$
m_{\leq k}\left(\Delta^{s}(G)\right) \leq \operatorname{dim}_{K}\left\{\phi_{g, k+1}^{n}(E(G))\right\}=\operatorname{dim}_{K}\left\{\varphi_{g, k+1}^{n}(E(G))\right\}=m_{\leq k+1}\left(\Delta^{e}(G)\right) .
$$

Next, we will prove the second inequality of (1). We use Kalai's idea in [6]. Define a linear map $f_{i}^{*}(A): V \rightarrow K$ by

$$
f_{i}^{*}(A)\left(e_{k}\right)= \begin{cases}x_{i k}, & \text { if } k \in A, \\ 0, & \text { if } k \notin A .\end{cases}
$$

For $i, j \in[n]$ we define $F_{k}(i, j): \bigoplus^{k} V \rightarrow K$ as follows:

For $v=\left(v_{1}, \ldots, v_{k}\right) \in \bigoplus^{k} V$, we define

$$
F_{k}(i, j)(v)=f_{i}^{*}(A)\left(v_{j}\right)-f_{j}^{*}(B)\left(v_{i}\right) .
$$

Let $F_{k}=\left(F_{k}(1,1), F_{k}(1,2), F_{k}(2,2), F(1,3), F(2,3), F(3,3), \ldots, F_{k}(k, k)\right)$, and let $W_{k}=\left\{v \in \bigoplus^{k} V \mid F_{k}(v) \neq 0\right\}$. For any edge $s \in K_{A, B}$, we have $F_{k}(i, j)\left(\phi_{g, k}^{n}(s)\right)=0$ for $1 \leq i, j \leq k$. Thus we have $\phi_{g, k}^{n}(E(G)) \cap W_{k}=\emptyset$. Then what we want to prove is

$$
\operatorname{dim}_{K}\left\{\operatorname{span}\left\{\phi_{g, k}^{n}(\{1,1\}), \ldots, \phi_{g, k}^{n}(\{n, n\})\right\}\right\} \cap W_{k}=\min \left\{\left(\begin{array}{c}
k+1 \\
2
\end{array}\right), n\right\} .
$$


Now, one has

$$
F_{k}\left(\phi_{g, k}^{n}(\{l, l\})=\left\{\begin{aligned}
\bigoplus_{1 \leq i \leq j \leq k}\left(x_{i l} x_{j l}\right), & \text { if } l \in A, \\
-\bigoplus_{1 \leq i \leq j \leq k}\left(x_{i l} x_{j l}\right), & \text { if } l \in B .
\end{aligned}\right.\right.
$$

Let $M_{g, k}$ be the $n \times\left(\begin{array}{c}k+1 \\ 2\end{array}\right)$ matrix whose columns are indexed by $(i, j)$ with $i \leq j$ and whose $(l,(i, j))$ th entry is $x_{i l} x_{j l}$, that is, rows of $M_{g, k}$ is corresponding with $F_{k}\left(\phi_{g, k}^{n}(\{l, l\})\right)$. Then $\operatorname{dim}_{K}\left\{\operatorname{span}\left\{\phi_{g, k}^{n}(\{1,1\}), \ldots, \phi_{g, k}^{n}(\{n, n\})\right\}\right\} \cap W_{k}=\operatorname{rank} M_{g, k}$. To prove $\operatorname{rank} M_{g, k}=\min \left\{\left(\begin{array}{c}k+1 \\ 2\end{array}\right), n\right\}$, we consider the following:

Every positive integer $l$ have a unique representation $l=\left(\begin{array}{c}h(l) \\ 2\end{array}\right)+\alpha(l)$ with $0<$ $\alpha(l) \leq h(l)$ and with $h(l) \geq 1$. Let $g^{\prime}=\left(y_{i j}\right) \in G L_{n}(K)$ which satisfies

$$
y_{j l}= \begin{cases}1, & \alpha(l) \leq j \leq h(l), \\ 0, & \text { else }\end{cases}
$$

Then the matrix $M_{g^{\prime}, k}$ is

$$
M_{g^{\prime}, k}=\left(\begin{array}{ccccc}
B_{1} & 0 & 0 & \cdots & 0 \\
* & B_{2} & 0 & \cdots & 0 \\
* & * & B_{3} & \cdots & 0 \\
\vdots & \vdots & \vdots & \ddots & \vdots \\
* & * & * & \cdots & B_{k}
\end{array}\right),
$$

where $B_{i}$ is $i \times i$ upper triangular matrix whose $(i, j)$ th entries with $i \leq j$ are 1 , that is

$$
B_{i}=\left(\begin{array}{cccc}
1 & 1 & \cdots & 1 \\
0 & 1 & \cdots & 1 \\
\vdots & \vdots & \ddots & \vdots \\
0 & 0 & \cdots & 1
\end{array}\right)
$$

Thus we have $\operatorname{rank} M_{g^{\prime}, k}=\min \left\{\left(\begin{array}{c}k+1 \\ 2\end{array}\right), n\right\}$. By the same argument of Lemma 1.2, we have $\operatorname{rank} M_{g, k} \geq \operatorname{rank} M_{g^{\prime} k}$. Since $\min \left\{\left(\begin{array}{c}k+1 \\ 2\end{array}\right), n\right\}$ is the maximal rank of $n \times\left(\begin{array}{c}k+1 \\ 2\end{array}\right)$ matrix, we have $\operatorname{rank} M_{g, k}=\min \left\{\left(\begin{array}{c}k+1 \\ 2\end{array}\right), n\right\}$. Hence

$$
\begin{aligned}
m_{\leq k-1}\left(\Delta^{s}(G)\right)+n & =\operatorname{dim}_{K}\left\{\phi_{g, k}^{n}(E(G)) \cup \operatorname{span}\left\{\phi_{g, k}^{n}(\{1,1\}), \ldots, \phi_{g, k}^{n}(\{n, n\})\right\}\right\} \\
& \left.\geq \operatorname{dim}_{K}\left\{\phi_{g, k}^{n}(E(G)) \cup \operatorname{span}\left\{\phi_{g, k}^{n}(\{1,1\}), \ldots, \phi_{g, k}^{n}(\{n, n\})\right\} \cap W_{k}\right\}\right\} \\
& =\operatorname{dim}_{K} \phi_{g, k}^{n}(E(G))+\operatorname{rank} M_{g, k} \\
& =\operatorname{dim}_{K} \phi_{g, k}^{n}(E(G))+\min \left\{\left(\begin{array}{c}
k+1 \\
2
\end{array}\right), n\right\} \\
& =m_{\leq k}\left(\Delta^{e}(G)\right)+\min \left\{\left(\begin{array}{c}
k+1 \\
2
\end{array}\right), n\right\},
\end{aligned}
$$

as desired.

By Lemma 2.2, we can determine the $\Delta^{s}\left(K_{A, B}\right)$. Before its proof we introduce some definitions and lemmas in [6]. 
Definition 2.3. Let $G$ be a graph on $[n]$.

(i) A graph $G$ is called $k$-hyperconnected if $\{k, n\} \in \Delta^{e}(G)$.

(ii) A graph $G$ is called generically $k$-rigid if $\{k, n\} \in \Delta^{s}(G)$.

For a geometrical meaning of rigidity, see $[7, \S 2.7]$. Kalai proved if $G$ is $k$-hyperconnected then $G$ is $k$-connected and if $G$ is generically $k$-rigid then $G$ is $k$-connected.

Lemma 2.4 ([6, Lemma 4.3 and $\S 8])$. Let $G$ be a graph on $[n-1]$, and let $G^{\prime} \supset G$ be a graph on $[n]$.

(i) If $G$ is $k$-hyperconnected (generically $k$-rigid) and $\operatorname{deg}(n) \geq k$ then $G^{\prime}$ is also $k$-hyperconnected (generically $k$-rigid).

(ii) If $\{k+1, k+2\} \notin \Delta^{e}(G)\left(\{k+1, k+2\} \notin \Delta^{s}(G)\right)$ and $\operatorname{deg}(n) \leq k$ then $\{k+1, k+2\} \notin \Delta^{e}(G)^{\prime}\left(\{k+1, k+2\} \notin \Delta^{s}(G)^{\prime}\right)$.

Kalai's proof is the case of $\Delta^{e}(G)$. But the case of $\Delta^{s}(G)$ can be proved by the same way.

Theorem 2.5. Let $G=K_{A, B}$ be the complete bipartite graph with $A \neq \emptyset, B \neq \emptyset$ and $|A|+|B|=n$. Let $n=\left(\begin{array}{c}h(n) \\ 2\end{array}\right)+\alpha(n)$ with $0<\alpha(n) \leq h(n)$ and $h(n) \geq 1$. Then $m_{\leq k}\left(\Delta^{s}(G)\right)= \begin{cases}\left(\begin{array}{c}n \\ 2\end{array}\right)-\left(\begin{array}{c}n-k \\ 2\end{array}\right), & \text { if } k \leq \min \{|A|,|B|\}-1 \text { and } k \leq h(n)-2, \\ (k+1) n-(k+1)^{2}, & \text { if } k \leq \min \{|A|,|B|\}-1 \text { and } k>h(n)-2, \\ |A||B|, & \text { if } k>\min \{|A|,|B|\}-1 .\end{cases}$

Proof. We use induction on $|A|-|B|$.

(i) Firstly, we assume $|A|=|B|$. Then $n=2|A|$ and $n \leq\left(\begin{array}{c}|A|+1 \\ 2\end{array}\right)$. Thus $h(n) \leq$ $|A|+1$. By Lemma 2.1, we have $m_{\leq k}\left(\Delta^{e}(G)\right)-n=\left(\begin{array}{l}n \\ 2\end{array}\right)-\left(\begin{array}{c}n-k+1 \\ 2\end{array}\right)-\left(\begin{array}{c}k+1 \\ 2\end{array}\right)$. Now, $m_{\leq k}\left(\Delta^{s}(G)\right)$ satisfies (1). Thus for $k \leq h(n)-2$ we have $m_{\leq k}\left(\Delta^{s}(G)\right) \geq$ $m_{\leq k+1}\left(\Delta^{e}(G)\right)+\left(\begin{array}{c}k+2 \\ 2\end{array}\right)=\left(\begin{array}{c}n \\ 2\end{array}\right)-\left(\begin{array}{c}n-k \\ 2\end{array}\right)$. But Lemma 1.3 says $m_{\leq k}\left(\Delta^{s}(G)\right) \leq\left(\begin{array}{c}n \\ 2\end{array}\right)-\left(\begin{array}{c}n-k \\ 2\end{array}\right)$. Thus we have $m_{\leq k}\left(\Delta^{s}(G)\right)=\left(\begin{array}{c}n \\ 2\end{array}\right)-\left(\begin{array}{c}n-k \\ 2\end{array}\right)$ for $k \leq h(n)-2$. If $k>h(n)-2$, then $\left(\begin{array}{c}k+2 \\ 2\end{array}\right) \geq n$. Thus (1) says $m_{\leq k}\left(\Delta^{s}(G)\right)=m_{\leq k+1}\left(\Delta^{e}(G)\right)$. Hence we have the equality which we want.

We assume $|A|<|B|$. Let $|A|=a$ and $B^{\prime} \subset B$ be the subset with $|B|-\left|B^{\prime}\right|=1$. (ii) If $\{a-1, n-1\} \in \Delta^{s}\left(K_{A, B^{\prime}}\right)$, then Lemma 2.4 says $\{a-1, n\} \in \Delta^{s}\left(K_{A, B}\right)$. Then, $n$ must satisfy $n>\left(\begin{array}{c}a+1 \\ 2\end{array}\right)$ and $h(n) \geq a+1$. Hence we have $m_{\leq k}\left(\Delta^{s}(G)\right)=\left(\begin{array}{c}n \\ 2\end{array}\right)-\left(\begin{array}{c}n-k \\ 2\end{array}\right)$ for $k \leq a-1 \leq h(n)-2$. Also, assumption of induction says $\{a+1, a+2\} \notin$ $\Delta^{s}\left(K_{A, B^{\prime}}\right)$. Thus we have $\{a+1, a+2\} \notin \Delta^{s}\left(K_{A, B}\right)$ by Lemma 2.4. Since $\Delta^{s}(G)$ is shifted, we have $m_{\leq k}\left(\Delta^{s}(G)\right)=|A||B|$ for $k \geq a$. Thus $\Delta^{s}\left(K_{A, B}\right)$ satisfies the equality which we want.

(iii) If $\{a-1, n-1\} \notin \Delta^{s}\left(K_{A, B^{\prime}}\right)$, then we have $h(n)<a+1$. By the same argument of (i) we have the equality.

Corollary 2.6. Let $G$ be a bipartite graph and let $n=\left(\begin{array}{c}h(n) \\ 2\end{array}\right)+\alpha(n)$ with $0<\alpha(n) \leq$ $h(n)$ and $h(n) \geq 1$. If $\{h(n), h(n)+1\} \in \Delta^{e}(G)$, then $\Delta^{e}(G) \neq \Delta^{s}(G)$. 
Proof. By Lemma 2.2 and $n \leq\left(\begin{array}{c}h(n)+1 \\ 2\end{array}\right)$, we have $m_{\leq h(n)-1}\left(\Delta^{s}(G)\right) \geq m_{\leq h(n)}\left(\Delta^{e}(G)\right)$. Since $\{h(n), h(n)+1\} \in \Delta^{e}(G)$, we have $m_{\leq h(n)}\left(\Delta^{e}(G)\right)>m_{\leq h(n)-1}\left(\Delta^{e}(G)\right)$. Thus we have $m_{\leq h(n)-1}\left(\Delta^{s}(G)\right)>m_{\leq h(n)-1}\left(\Delta^{e}(G)\right)$.

Example 2.7. By Lemma 2.1 and Theorem 2.5, we can easily compute $\Delta^{e}\left(K_{A, B}\right)$ and $\Delta^{s}\left(K_{A, B}\right)$. For example,

$\Delta^{e}\left(K_{6,6}\right)=\{\{1,2\}, \ldots,\{1,12\},\{2,3\}, \ldots,\{2,11\},\{3,4\}, \ldots,\{3,10\}$,

$\{4,5\}, \ldots,\{4,9\},\{5,6\}, \ldots,\{5,8\},\{6,7\}\}$

and

$\Delta^{s}\left(K_{6,6}\right)=\{\{1,2\}, \ldots,\{1,12\},\{2,3\}, \ldots,\{2,12\},\{3,4\}, \ldots,\{3,12\}$, $\{4,5\}, \ldots\{4,9\},\{5,6\}\}$.

Also, Corollary 2.6 implies $\Delta^{e}(G) \neq \Delta^{s}(G)$ for many of the bipartite graphs $G$. If $G$ is bipartite graph on $[n]$ and $|G|>\left(\begin{array}{l}n \\ 2\end{array}\right)-\left(\begin{array}{c}n-h(n)+1 \\ 2\end{array}\right)$, then $\{h(n), h(n)+1\} \in \Delta^{e}(G)$. Thus Corollary 2.6 implies $\Delta^{e}(G) \neq \Delta^{s}(G)$. For example, if $G \subset K_{50,50}$ have more than 1000 edges, then $\Delta^{e}(G) \neq \Delta^{s}(G)$. Note $\left|K_{50,50}\right|=2500$.

One interesting problem of algebraic shifting is differences between $\Delta^{e}(G)$ and $\Delta^{s}(G)$. For $\Delta^{c}(G)$ and $\Delta^{e}(G)$, we proved $m_{\leq k}\left(\Delta^{c}(G)\right) \leq m_{\leq k}\left(\Delta^{e}(G)\right)$ in [11]. So inequalities $m_{<k}\left(\Delta^{e}(G)\right) \leq m_{\leq k}\left(\Delta^{s}(G)\right)$ are tempting conjecture. They are also related to the Herzog's conjecture about graded Betti numbers that we see later in $\S 4$. We only know the followings.

Proposition 2.8. Let $G$ be a graph on $[n]$. Then one has

(i) $m_{\leq 1}\left(\Delta^{e}(G)\right)=m_{\leq 1}\left(\Delta^{s}(G)\right)$,

(ii) $m_{\leq 2}\left(\Delta^{e}(G)\right) \leq m_{\leq 2}\left(\Delta^{s}(G)\right)$.

Proof. Recall $\varphi_{g, 1}^{n}(\{i, j\})=x_{1 j} e_{i}-x_{1 i} e_{j}$. Let $e_{k}=x_{1 k} e_{k}^{\prime}$. Then $\varphi_{g, 1}^{n}(\{i, j\})=$ $x_{1 j} x_{1 i}\left(e_{i}-e_{j}\right)$. We may assume $x_{1 j} x_{1 i} \neq 0$ and $\varphi_{g, 1}^{n}=\left(e_{i}-e_{j}\right)$. On the other hand, $\rho_{g, 1}^{n}(\{i, j\})=\left(x_{1 i}-x_{1 j}\right)\left(e_{i}-e_{j}\right)$. Thus we may assume $\rho_{g, 1}^{n}(\{i, j\})=\left(e_{i}-e_{j}\right)$. In this case $\varphi_{g, 1}^{n}=\rho_{g, 1}^{n}$. Hence $m_{\leq 1}\left(\Delta^{e}(G)\right)=m_{\leq 1}\left(\Delta^{s}(G)\right)$.

Next by the same argument, we may assume $\varphi_{g, 2}^{n}=\left(e_{i}-e_{j}, x_{2 j} e_{i}-x_{2 i} e_{j}\right)$. Let $B=\left\{(0, f) \in \varphi_{g, 2}^{n}(E(G))\right\} \subset \varphi_{g, 2}^{n}(E(G))$. Then we have

$$
\operatorname{dim} \varphi_{g, 2}^{n}(E(G))=\operatorname{dim} \varphi_{g, 1}^{n}(E(G))+\operatorname{dim} B .
$$

On the other hand, $\rho_{g, 2}^{n}(\{i, j\})=\left(\left(x_{1 i}-x_{1 j}\right)\left(e_{i}-e_{j}\right),\left(x_{2 i}-x_{2 j}\right)\left(e_{i}-e_{j}\right)\right)$. Let $\rho_{g^{\prime}, 2}^{n}(\{i, j\})=\left(e_{i}-e_{j},\left(x_{2 i}+x_{2 j}\right)\left(e_{i}-e_{j}\right)\right) \cdot \rho_{g^{\prime}, 2}^{n}$ is given by the substitution $x_{1 k}=x_{2 k}$ and $x_{2 k}=x_{2 k}^{2}$. Since substitutions reduce the dimension by the same argument of Lemma 1.2, we have

$$
\operatorname{dim} \rho_{g, 2}^{n}(E(G)) \geq \operatorname{dim} \rho_{g^{\prime}, 2}^{n}(E(G)) .
$$

Let $C=\left\{(0, f) \in \rho_{g^{\prime}, 2}^{n}(E(G))\right\} \subset \rho_{g^{\prime}, 2}^{n}(E(G))$. Then

$$
\operatorname{dim} \rho_{g^{\prime}, 2}^{n}(E(G))=\operatorname{dim} \rho_{g^{\prime}, 1}^{n}(E(G))+\operatorname{dim} C .
$$

We will prove $\operatorname{dim} B=\operatorname{dim} C . B$ and $C$ are generated by a cycle in $E(G)$, that is, $B=\operatorname{span}\left\{\sum_{k=1}^{r} \varphi_{g, 2}^{n}\left(\left\{a_{k}, a_{k+1}\right\}\right) \mid\left(\left\{a_{1}, a_{2}\right\}, \ldots,\left\{a_{r}, a_{r+1}\right\}\right)\right.$ is a cycle in $\left.E(G)\right\}$. In this case $\sum_{k=1}^{r} \varphi_{g, 2}^{n}\left(\left\{a_{k}, a_{k+1}\right\}\right)=\left(0, \sum_{k=1}^{r}\left(x_{2 a_{k+1}}-x_{2 a_{k-1}}\right) e_{a_{k}}\right)$, where $a_{0}=a_{r}$ 
and $a_{r+1}=a_{1}$. Also, we have $\sum_{k=1}^{r} \rho_{g^{\prime}, 2}^{n}\left(\left\{a_{k}, a_{k+1}\right\}\right)=\left(0, \sum_{k=1}^{r}\left(x_{2 a_{k+1}}-x_{2 a_{k-1}}\right) e_{a_{k}}\right)$. Thus we have $\operatorname{dim} B=\operatorname{dim} C$. By (5), (6) and (7), we have

$m_{\leq 2}\left(\Delta^{s}(G)\right)=\operatorname{dim} \rho_{g, 2}^{n}(E(G)) \geq \operatorname{dim} \rho_{g^{\prime}, 2}^{n}(E(G))=\operatorname{dim} \varphi_{g, 2}^{n}(E(G))=m_{\leq 2}\left(\Delta^{e}(G)\right)$,

as desired.

\section{Generic initial ideals}

In this section we will introduce ring theoretical definition of exterior shifting and symmetric shifting in [8]. Let $K$ be the infinite field with $\operatorname{char}(K)=0, R=$ $K\left[x_{1}, x_{2}, \ldots, x_{n}\right]$ the polynomial ring, $\wedge^{*} V$ the exterior algebra. Let $\operatorname{gin}(I)$ denote a generic initial ideal of homogeneous ideal $I \subset R$ and $\operatorname{Gin}(J)$ a generic initial ideal of homogeneous ideal $J \subset \bigwedge^{*} V$. We refer the reader to [8] for the definition and further properties of generic initial ideals. Let $I \subset R$ be a monomial ideal with a minimal set of monomial generators $G(I)$ whose elements $u=x_{i_{1}} x_{i_{2}} \cdots x_{i_{d}} \in G(I)$ satisfies $i_{j} \geq i_{j+1}$ and $i_{d}>d-1$. Then $\rho(u)=x_{i_{1}} x_{i_{2}-1} \cdots x_{i_{d}-(d-1)}$ is a squarefree monomial. Let $\rho(I)$ be an ideal generated by $\rho(G(I))=\{\rho(u) \mid u \in G(I)\}$.

Let $G$ be a graph, $I_{G} \subset R$ the Stanley-Reisner ideal, in other words, $I_{G}$ is an ideal generated by $\left\{x_{i} x_{j} \mid\{i, j\} \notin E(G)\right\}$ and all squarefree monomials with degree 3. Let $J_{G} \subset \wedge^{*} V$ be an ideal generated by $\left\{e_{i} \wedge e_{j} \mid\{i, j\} \notin E(G)\right\}$ and all squarefree monomials with degree 3 . Let $\tilde{I}_{G} \subset R$ be an ideal generated by $\left\{x_{i} x_{j} \mid\{i, j\} \notin E(G)\right\}$. $\Delta^{e}(G)$ and $\Delta^{s}(G)$ can be described by using generic initial ideal.

Lemma $3.1([8, \S 8])$. We consider the reverse lexicographic order with $x_{1}<x_{2}<$ $\cdots<x_{n}$. Then one has

(i) $J_{\Delta^{e}(G)}=\operatorname{Gin}\left(J_{G}\right)$,

(ii) $I_{\Delta^{s}(G)}=\rho\left(\operatorname{gin}\left(I_{G}\right)\right)$.

Next, we introduce relations between graded Betti numbers and algebraic shifting. We call $\beta_{i j}(I)=\operatorname{dim}_{K} \operatorname{Tor}_{i}(K, I)_{j}$ the graded Betti numbers of $I$. Betti numbers of $\tilde{I}_{G}$ are easily computed if $G$ is shifted. We note one of its form ([11, Lemma 3.1]).

$\beta_{i i+j}\left(\tilde{I}_{G}\right)=m_{\leq n}\left(\tilde{I}_{G}, j\right)\left(\begin{array}{c}n-j \\ i\end{array}\right)-\sum_{k=j}^{n-1} m_{\leq k}\left(\tilde{I}_{G}, j\right)\left(\begin{array}{c}k-j \\ i-1\end{array}\right)-\sum_{k=j}^{n} m_{\leq k-1}\left(\tilde{I}_{G}, j-1\right)\left(\begin{array}{c}k-j \\ i\end{array}\right)$,

where $m_{\leq k}(I, j)$ denote the number of squarefree monomial $u \in I$ with $\operatorname{deg}(u)=$ $j$ and with $\max \left\{i \in[n] \mid x_{i}\right.$ divides $\left.u\right\} \leq k$. This equality implies graded Betti numbers determine $m_{\leq k}(G)$, therefore, determine a shifted graph $G$. Thus we have the following lemma.

Lemma 3.2. Let $G$ and $G^{\prime}$ be the shifted graph. Then the followings are equivalent.

(i) $\beta_{i j}\left(\tilde{I}_{G}\right)=\beta_{i j}\left(\tilde{I}_{G^{\prime}}\right)$ for all $i, j$;

(ii) $m_{\leq k}(G)=m_{\leq k}\left(G^{\prime}\right)$ for all $k$;

(iii) $G=G^{\prime}$. 
Let $I$ be a homogeneous ideal generated in one degree $d$. We say $I$ has a linear resolution if $\beta_{i i+j}(I)=0$ for $j \neq d$. Let $G$ be a graph and $\left(\left\{r_{1}, r_{2}\right\}, \ldots,\left\{r_{s}, r_{1}\right\}\right)$ a cycle in $G$. An edge $\{i, j\}$ is called a chord of cycle $\left(\left\{r_{1}, r_{2}\right\}, \ldots,\left\{r_{s}, r_{1}\right\}\right)$ if $i, j \in\left\{r_{1}, r_{2}, \ldots, r_{s}\right\}$ and $\{i, j\} \neq\left\{r_{i}, r_{i+1}\right\}$ for all $i$. We call $G$ chordal if every cycle of length $>3$ has a chord. The following properties are known.

Lemma 3.3. Let $G$ be the finite graph. Then the followings are equivalent.

(i) $G$ is chordal;

(ii) $\tilde{I}_{G}$ has a linear resolution;

(iii) $I_{G}, I_{\Delta^{e}(G)}$ and $I_{\Delta^{s}(G)}$ have same graded Betti numbers.

Proof. (i) $\Leftrightarrow\left(\right.$ ii) is in [5]. $\tilde{I}_{G}$ has a linear resolution if and only if $\beta_{i j}\left(\operatorname{gin}\left(I_{G}\right)\right)=\beta_{i j}\left(I_{G}\right)$ and $\beta_{i j}\left(I_{\Delta e}(G)\right)=\beta_{i j}\left(I_{G}\right)$ ([2, Theorem 2.1 and Corollary 2.3]). On the other hand, $\beta_{i j}\left(\operatorname{gin}\left(I_{G}\right)\right)=\beta_{i j}\left(\rho\left(\operatorname{gin}\left(I_{G}\right)\right)\right)([8$, Lemma 8.18]). Thus we have (ii) $\Leftrightarrow($ iii).

This and Lemma 3.2 say $\Delta^{e}(G)=\Delta^{s}(G)$ for any chordal graph $G$.

\section{Algebraic shifting of Chordal graphs}

Firstly, we introduce a combinatorial shifting $\Delta^{c}$. Erdös, Ko and Rado [4] introduce a combinatorial shifting. Let $G$ be a finite graph on $[n]$. Let $1 \leq i<j \leq n$. Write $\operatorname{Shift}_{i j}(G)$ for the graph on $[n]$ whose edges are $C_{i j}(s) \subset[n]$, where $s \in G$ and where

$$
C_{i j}(s)= \begin{cases}(s \backslash\{i\}) \cup\{j\}, & \text { if } i \in s, j \notin s \text { and }(s \backslash\{i\}) \cup\{j\} \notin G, \\ \sigma, & \text { otherwise. }\end{cases}
$$

Note that Shift $_{i j}$ only depends on the choice of $i$ and $j$, that is $\operatorname{Shift}_{i j}(G) \simeq$ $\operatorname{Shift}_{j i}(G)$. It follows from, e.g., [8, Corollary 8.6] that there exists a finite sequence of pairs of integers $\left(i_{1}, j_{1}\right),\left(i_{2}, j_{2}\right), \ldots,\left(i_{q}, j_{q}\right)$ with each $1 \leq i_{k}<j_{k} \leq n$ such that

$$
\operatorname{Shift}_{i_{q} j_{q}}\left(\operatorname{Shift}_{i_{q-1} j_{q-1}}\left(\cdots\left(\operatorname{Shift}_{i_{1} j_{1}}(G)\right) \cdots\right)\right)
$$

is shifted. Such a shifted graph is called a combinatorial shifted graph of $G$ and will be denoted by $\Delta^{c}(G)$. A combinatorial shifted graph $\Delta^{c}(G)$ is, however, not necessarily unique. Combinatorial shifting has a bad behavior. But it is easy to compute.

Let $G=(V, E(G))$ be a graph on the vertex set $V$. We call $v \in V$ a central vertex if $G$ satisfies $\{u, v\} \in E(G)$ or $\operatorname{deg}(u)=0$ for any $u \in V \backslash\{v\}$. Let $G \backslash\{v\}$ be the induced subgraph on $V \backslash\{v\}$. Central vertices do not affect $\Delta^{c}(G)$ (same property is true for $\Delta^{e}(G)$ and $\left.\Delta^{s}(G)\right)$.

Lemma 4.1. Let $G=([n], E(G))$ be a graph with a central vertex $u$ and $\operatorname{deg}(u)=h$. Then $\Delta^{c}(G)=\Delta^{c}(G \backslash\{u\})_{[2, n]} \cup\{\{1,2\}, \ldots,\{1, h+1\}\}$, where $\Delta^{c}(G \backslash\{u\})_{[2, n]}$ is a combinatorial shifted graph on $[2, n]=\{2, \ldots, n\}$.

Proof. We may assume $u=1$. Then $\operatorname{Shift}_{1 j}(G)=G$ for any $j \in[n]$. Thus we may only consider a combinatorial shifting of $G \backslash\{u\}$. 
Let $G$ be a graph with connected components $A_{1}, \ldots, A_{r}$. In general, we can easily show $m_{1}\left(\Delta^{c}(G)\right) \leq n-r=m_{1}\left(\Delta^{e}(G)\right)$. But we can make a $\Delta^{c}(G)$ such that $m_{1}\left(\Delta^{c}(G)\right)=m_{1}\left(\Delta^{e}(G)\right)$ by the following way.

Lemma 4.2. Let $G=([n], E(G))$ be a connected graph. Then there is $\Delta^{c}(G)$ such that $\{1, n\} \in \Delta^{c}(G)$.

Proof. Since $G$ is connected, if vertex 1 is not a central vertex, then there is $\{1, v\} \in$ $E(G)$ such that $\{u, v\} \in E(G)$ and $\{u, 1\} \notin E(G)$. Then $\{u, 1\} \in \operatorname{Shift}_{1 v}(E(G))$. We will prove $\operatorname{Shift}_{1 v}(E(G))$ is also connected.

Since $G$ is connected, any vertex $w$ has a path to 1 . Let $\left(\left\{1, r_{1}\right\},\left\{r_{1}, r_{2}\right\}, \ldots,\left\{r_{s}, w\right\}\right)$ be a path.

If $r_{k} \neq v$ for any $k$, then $\operatorname{Shift}_{1 v}(E(G))$ has the same path.

If $r_{k}=v$ for some $k$, then $\left\{1, r_{k+1}\right\} \in \operatorname{Shift}_{1 v}(E(G))$. Thus $\operatorname{Shift}_{1 v}(E(G))$ has a path from 1 to $w$. Inductively, we can create a central vertex. Then Lemma 4.1 says $\{1, n\} \in \Delta^{c}(G)$ for some $\Delta^{c}(G)$.

Next, we find all chordal graphs whose combinatorial shifting is unique. Firstly we introduce an example whose combinatorial shifting is unique.

Lemma 4.3. Let $G=K_{A} \cup K_{B}$ be a disjoint union of two complete graphs $K_{A}$ and $K_{B}$ with $|A|=a$ and $|B|=b$. Then $\Delta^{c}(G)$ is unique and

$$
m_{\leq k}\left(\Delta^{c}(G)\right)=\left(\begin{array}{l}
a \\
2
\end{array}\right)-\left(\begin{array}{c}
a-k \\
2
\end{array}\right)+\left(\begin{array}{l}
b \\
2
\end{array}\right)-\left(\begin{array}{c}
b-k \\
2
\end{array}\right) .
$$

Proof. If $i \in A$ and $j \in A$, then $\operatorname{Shift}_{i j}(G)=G$. Thus we may assume $i \in A, j \in B$ and $i<j$. Then $i$ is a central vertex on $H=\operatorname{Shift}_{i j}(G)$. Also, $H \backslash\{i\}$ is a disjoint union of $K_{A \backslash\{i\}}$ and $K_{B \backslash\{j\}}$. Inductively, we get a result.

[Remark]. Complementary graph of complete bipartite graph is a disjoint union of two complete graph. Thus $\Delta^{c}\left(K_{A, B}\right)$ is unique and $\Delta^{e}\left(K_{A, B}\right)=\Delta^{c}\left(K_{A, B}\right)$ follows from Lemma 4.7.

Proposition 4.4. Let $G=([n], E(G))$ be a chordal graph whose combinatorial shifting is unique. Then there are integers $r$ and $n_{1} \geq n_{2} \geq \cdots \geq n_{r} \geq 0$ such that

$$
E(G)=\left\{\left\{i, j_{i}\right\} \mid 1 \leq i \leq r, i+1 \leq j_{i} \leq n_{i}\right\} \cup K_{A} \cup K_{B},
$$

where $K_{A} \cup K_{B}$ is the disjoint union of complete graphs with $A \cup B \subset\left\{r+1, \ldots, n_{r}\right\}$.

Proof. We may assume $G$ has no isolated vertices, since isolated vertices do not affect $\Delta^{c}(G)$.

[Case 1] Let $G$ have 3 connected components $A_{1}, A_{2}, A_{3}$, and let $a_{1}, b_{1} \in A_{1}, a_{2}, b_{2} \in$ $A_{2}$ and $a_{3}, b_{3} \in A_{3}$. We may assume $a_{1}<b_{2}, a_{2}<b_{3}$ and $a_{3}<b_{1}$. Let $H_{1}=$ Shift $_{a_{2} b_{3}}\left(\operatorname{Shift}_{a_{1} b_{2}}(G)\right)$ and $H_{2}=\operatorname{Shift}_{a_{3} b_{1}}\left(H_{1}\right)$. Then $\operatorname{deg}\left(b_{1}\right)=\operatorname{deg}\left(b_{2}\right)=\operatorname{deg}\left(b_{3}\right)=$ 0 on $H_{2}$. Thus $m_{1}\left(\Delta^{c}\left(H_{2}\right)\right) \leq n-4$. Since $H_{1}$ is connected, there is $\Delta^{c}\left(H_{1}\right)$ such that $m_{1}\left(\Delta^{c}\left(H_{1}\right)\right)=n-3$. This contradicts a uniqueness of $\Delta^{c}(G)$. By the same argument, if $G$ has more than 3 connected components, then $\Delta^{c}(G)$ is not unique. 
[Case 2] Let $G$ have 2 connected components $A$ and $B$. If $G=K_{A} \cup K_{B}$, then $\Delta^{c}(G)$ is unique. Assume there are $a_{1}, a_{2} \in A$ such that $\left\{a_{1}, a_{2}\right\} \notin E(G)$. Take any vertex $b_{1}, b_{2} \in B$. Assume $a_{1}<b_{1}$ and $a_{2}<b_{2}$. Let $H_{1}=\operatorname{Shift}_{a_{1} b_{1}}(G)$ and $H_{2}=\operatorname{Shift}_{a_{2} b_{2}}\left(H_{1}\right)$. Then for any $\left\{p, a_{2}\right\} \in E\left(H_{1}\right)$ we have $p \in A \backslash\left\{a_{1}\right\}$ and for any $\left\{q, b_{2}\right\} \in E\left(H_{1}\right)$ we have $q \in B \cup\left\{a_{1}\right\}$. Thus $\operatorname{deg}\left(b_{1}\right)=\operatorname{deg}\left(b_{2}\right)=0$ on $H_{2}$, therefore, $m_{1}\left(\Delta^{c}\left(H_{2}\right)\right) \leq n-3$. Since $H_{1}$ is connected, there is $\Delta^{c}\left(H_{1}\right)$ such that $m_{1}\left(\Delta^{c}\left(H_{1}\right)\right)=n-2$. This contradicts the uniqueness of $\Delta^{c}(G)$.

[Case 3] Let $G$ be a connected graph. Assume $G$ is not complete graph and $\{i, j\} \notin$ $E(G)$. Firstly, we separate vertices. Let

$$
\begin{aligned}
& A=\{p \in[n] \mid\{i, p\} \in E(G),\{j, p\} \in E(G)\}, \\
& X_{i}=\{p \in[n] \mid\{i, p\} \in E(G),\{j, p\} \notin E(G)\}, \\
& X_{j}=\{p \in[n] \mid\{i, p\} \notin E(G),\{j, p\} \in E(G)\}, \\
& X_{0}=\{p \in[n] \mid\{i, p\} \notin E(G),\{j, p\} \notin E(G)\} \text { and } X=X_{i} \cup X_{j} \cup X_{0} .
\end{aligned}
$$

[Step 1] We will prove $K_{A} \subset G$. For any vertices $a_{1}, a_{2} \in A, G$ has a cycle $\left(\left\{i, a_{1}\right\},\left\{a_{1}, j\right\},\left\{j, a_{2}\right\},\left\{a_{2}, i\right\}\right)$ with $\{i, j\} \notin E(G)$. Since $G$ is chordal, we have $\left\{a_{1}, a_{2}\right\} \in E(G)$ and $K_{A} \subset G$.

[Step 2] We will prove there is a vertex $a_{0} \in A$ such that $a_{0}$ is a central vertex of $G$. By [Step1], what we must prove is there is $a_{0} \in A$ such that $\left\{a_{0}, x\right\} \in E(G)$ for all $x \in X$. Let $A=\left\{a_{1}, a_{2}, \ldots, a_{r}\right\}$, and let $H_{1}=\operatorname{Shift}_{i j}(G)$. Assume $v<j$ for every vertex $v \in X$. Then for every $\{p, j\} \in E\left(H_{1}\right)$ we have $p \in A$ because of the definition of $X_{j}$.

Assume $a_{k}$ is not a central vertex for all $a_{k} \in A$, i.e. for any $a_{k} \in A$ there is $x_{a_{k}} \in X$ such that $\left\{a_{k}, x_{a_{k}}\right\} \notin G$. Let

$$
H_{2}=\operatorname{Shift}_{x_{a_{1}} j}\left(\operatorname{Shift}_{x_{a_{2} j}}\left(\cdots\left(\operatorname{Shift}_{x_{a_{r}} j}\left(H_{1}\right)\right) \cdots\right)\right) \text {. }
$$

Then $\operatorname{deg}(j)=0$ on $H_{2}$, since $\left\{a_{k}, j\right\} \notin E\left(H_{2}\right)$ by $\operatorname{Shift}_{x_{k} j}$ for $k=1,2, \ldots, r$. Thus $m_{1}\left(\Delta^{c}(G)\right) \leq n-2$, therefore, Lemma 4.2 says $\Delta^{c}(G)$ is not unique. Hence $G$ has a central vertex. Since $G$ has a central vertex $a_{0}$, we may only consider $G \backslash\left\{a_{0}\right\}$ by Lemma 4.1. On the other hand, $G \backslash\left\{a_{0}\right\}$ is chordal and has a unique combinatorial shifted graph. Hence, inductively we have the form of this lemma.

Let $I$ be a squarefree monomial ideal generated in one degree, $G(I)$ the minimal set of monomial generator of $I$. Let $G(I)^{\text {sqlex }}$ denote the squarefree lexsegment monomials with $|G(I)|=\left|G(I)^{\text {sqlex }}\right|, I^{\text {sqlex }}$ the squarefree monomial ideal generated by $G(I)^{\text {sqlex }}$. We refer the reader to [8] for the definition of segment ideals. We call $I$ is a squarefree Gotzmann monomial ideal if $I$ and $I^{\text {sqlex }}$ have the same Hilbert function.

Since we are interested in the classification of squarefree Gotzmann monomial ideal, we classify squarefree Gotzmann monomial ideals of degree 2 . Let $I$ be the squarefree monomial ideal generated in degree 2. Then there is a unique graph $G$ with $\tilde{I}_{G}=I$. Thus we consider a graph $G$. 
Corollary 4.5. Let $G$ be a graph that $\tilde{I}_{G}$ is a squarefree Gotzmann monomial ideal. Then $G$ is a squarefree revlexsegment or $G=K_{A} \cup K_{B}$ with $|A|=2$ and $A \cap B=\emptyset$.

Proof. Squarefree Gotzmann ideal have a linear resolution ([1, Theorem4.5]). Thus $G$ is chordal. If $I$ has a linear resolution, then its graded Betti numbers are determined by its Hilbert function. Thus $\tilde{I}_{G}$ and $\tilde{I}_{G}^{\text {sqlex }}$ have the same graded Betti numbers. By Lemmas 3.3 and 3.2, $\Delta^{e}(G)$ is a squarefree revlexsegment. Thus $I_{\Delta^{e}(G)}$ is a squarefree lexsegment ideal. Squarefree lexsegment ideal have a maximal graded Betti numbers among all squarefree monomial ideals with same Hilbert function ([8, Theorem 10.4]). Also, one has $\beta_{i j}\left(I_{\Delta^{c}(G)}\right) \geq \beta_{i j}\left(I_{\Delta^{e}(G)}\right)$ for all $i, j$ ([11, Theorem 3.3]). Since $I_{\Delta^{c}(G)}$ and $I_{\Delta^{e}(G)}$ have a same Hilbert function, we have $\beta_{i j}\left(I_{\Delta^{c}(G)}\right)=\beta_{i j}\left(I_{\Delta^{e}(G)}\right)$, therefore, Lemma 3.2 says $\Delta^{c}(G)$ is unique and $\Delta^{c}(G)=\Delta^{e}(G)$ is a squarefree revlexsegment. By Proposition 4.4 , we see $G$ must be a squarefree revlexsegment or $G=K_{A} \cup K_{B}$ with $|A|=2$ and $A \cap B=\emptyset$ if $\Delta^{c}(G)$ is a revlexsegment.

Secondly, we give an algorithm to compute exterior shifting and symmetric shifting of chordal graph $G$ using combinatorial shifting. For algorithm we use two types of shifting.

Let $G$ be a graph. We call $\operatorname{Shift}_{i j}(G)$ an edge shift if $\{i, j\} \in E(G)$.

Let $G$ be a graph with connected components $A_{1}, A_{2}, \ldots, A_{r}$ which are not isolated vertices. If $a_{i} \in A_{i}, a_{j} \in A_{j}$ and $i \neq j$, then we call $\operatorname{Shift}_{a_{i} a_{j}}(G)$ a disjoint shift.

Let $H\left(\tilde{I}_{G}, d\right)$ denote a $d$-th Hilbert function of $\tilde{I}_{G}$.

Lemma 4.6. Let $G$ be a chordal graph on $[n]$, and let $\operatorname{Shift}_{i j}(G)$ be an edge shift.

(i) $\operatorname{Shift}_{i j}(G)$ is also chordal.

(ii) $H\left(\tilde{I}_{G}, d\right)=H\left(\tilde{I}_{\text {Shift }_{i j}(G)}, d\right)$.

(iii) If $G$ is $k$-connected, then $\operatorname{Shift}_{i j}(G)$ is also k-connected.

Proof. Firstly we note the basic property of Shift ${ }_{i j}$.

$(\#)$ If $\{p, j\} \in E\left(\operatorname{Shift}_{i j}(G)\right)$ then $\{p, i\} \in E\left(\operatorname{Shift}_{i j}(G)\right) \cap E(G)$ and $\{p, j\} \in E(G)$.

(i) Assume a cycle $\left(\left\{r_{0}, r_{1}\right\},\left\{r_{1}, r_{2}\right\}, \ldots,\left\{r_{s}, r_{0}\right\}\right)$ in $\operatorname{Shift}_{i j}(G)$ does not have a chord and $s \geq 3$. Then definition of $\operatorname{Shift}_{i j}(G)$ says $i \in\left\{r_{0}, \ldots, r_{s}\right\}$ or $j \in$ $\left\{r_{0}, \ldots, r_{s}\right\}$. Also, since a cycle $\left(\left\{r_{0}, r_{1}\right\},\left\{r_{1}, r_{2}\right\}, \ldots,\left\{r_{s}, r_{0}\right\}\right)$ of $\operatorname{Shift}_{i j}(G)$ does not have a chord, we have

$$
\left\{r_{k}, r_{l}\right\} \notin E(G) \text { if } 1<|k-l|<s, r_{k} \notin\{i, j\} \text { and } r_{l} \notin\{i, j\} .
$$

[Case 1] If $i \in\left\{r_{0}, \ldots, r_{s}\right\}$, then by (\#) we may assume $j \notin\left\{r_{0}, \ldots, r_{s}\right\}$. Let $r_{0}=i$. Then a cycle $\left(\left\{i, r_{1}\right\},\left\{r_{1}, r_{2}\right\}, \ldots,\left\{r_{s}, i\right\}\right)$ or $\left(\left\{j, r_{1}\right\},\left\{r_{1}, r_{2}\right\}, \ldots,\left\{r_{s}, j\right\}\right)$ or $\left(\{i, j\},\left\{j, r_{1}\right\},\left\{r_{1}, r_{2}\right\}, \ldots,\left\{r_{s}, i\right\}\right)$ is in $G$. In each case, chordalness and (8) says these cycles must have a chord $\left\{i, r_{k}\right\}$ or $\left\{j, r_{k}\right\}$ for all $k$. Hence we have $\left\{i, r_{k}\right\} \in E\left(\operatorname{Shift}_{i j}(G)\right)$ for all $1 \leq k \leq s$. This is a contradiction.

[Case 2] If $j \in\left\{r_{0}, \ldots, r_{s}\right\}$, then we assume $r_{0}=j$. By the property (\#), we have $\left(\left\{j, r_{1}\right\},\left\{r_{1}, r_{2}\right\}, \ldots,\left\{r_{s}, j\right\}\right)$ and $\left(\left\{i, r_{1}\right\},\left\{r_{1}, r_{2}\right\}, \ldots,\left\{r_{s}, i\right\}\right)$ are cycle of $G$. Then chordalness and (8) say each cycle have a chord $\left\{i, r_{k}\right\}$ and $\left\{j, r_{k}\right\}$ for all 
$k$. Thus $\left\{i, r_{k}\right\} \in E(G)$ and $\left\{j, r_{k}\right\} \in E(G)$ for all $k$. This contradicts that $\left(\left\{j, r_{1}\right\},\left\{r_{1}, r_{2}\right\}, \ldots,\left\{r_{s}, j\right\}\right)$ is a cycle without a chord in $\operatorname{Shift}_{i j}(G)$.

(ii) Since $\tilde{I}_{G}$ is squarefree monomial ideal, $H\left(\tilde{I}_{G}, d\right)$ is determined by the number of squarefree monomial in $\tilde{I}_{G}\left(\left[3\right.\right.$, Theorem 5.1.7]). Let $x^{\sigma}=x_{i_{1}} x_{i_{2}} \cdots x_{i_{s}}$ denote a squarefree monomial with $\sigma=\left\{i_{1}, i_{2}, \ldots, i_{s}\right\} \subset[n]$. Let $D(d)=\{\sigma \subset[n]|| \sigma \mid=$ $\left.d, x^{\sigma} \notin \tilde{I}_{G}\right\}$. Then $D(d)$ can be described by $D(d)=\left\{\sigma \subset[n]|| \sigma \mid=d, K_{\sigma} \subset G\right\}$. Let $S(d)=\left\{\sigma \subset[n]|| \sigma \mid=d, K_{\sigma} \in \operatorname{Shift}_{i j}(G)\right\}$. Then $|D(d)|$ and $|S(d)|$ determine a Hilbert function of $\tilde{I}_{G}$ and $\tilde{I}_{\operatorname{Shift}_{i j}(G)}$. We will prove $S(d)=\operatorname{Shift}_{i j}(D(d))$, where $\operatorname{Shift}_{i j}(D(d))$ is defined by the same way of the graph case. $S(d) \supset \operatorname{Shift}_{i j}(D(d))$ is obvious. We will prove $S(d) \subset \operatorname{Shift}_{i j}(D(d))$. Let $\sigma \in S(d)$.

If $\sigma \in D(d)$, then $j \notin \sigma$ or $i, j \in \sigma$ or $\sigma \backslash\{j\} \cup\{i\} \in D(d)$. Thus $\sigma \in \operatorname{Shift}_{i j}(D(d))$. If $\sigma \notin D(d)$, we may assume $i \in \sigma$ and $j \notin \sigma$. We will prove $\sigma \backslash\{i\} \cup\{j\} \in D(d)$. Since $\sigma \notin D(d)$ and $\sigma \in S(d)$, there is $p \in \sigma \backslash\{i\}$ such that $\{i, p\} \notin E(G)$ and $\{i, p\} \in E\left(\operatorname{Shift}_{i j}(G)\right)$. Then $\{j, p\} \in E(G)$. For any $q \in \sigma \backslash\{i, p\}$, we have $\{q, i\} \in$ $E(G)$ or $\{q, j\} \in E(G)$. But if $\{q, i\} \in E(G)$ then $(\{j, p\},\{p, q\},\{q, i\},\{i, j\})$ is a cycle, therefore, we have $\{j, p\} \in E(G)$ by chordalness. Thus $\{q, j\} \in E(G)$ for any $q \in \sigma$. Since $K_{\sigma \backslash\{i\}} \subset G$, we have $\sigma \backslash\{i\} \cup\{j\} \in D(d)$.

Then we have $S(d)=\operatorname{Shift}_{i j}(D(d))$. Hence $|S(d)|=\left|\operatorname{Shift}_{i j}(D(d))\right|=|D(d)|$ and $H\left(I_{G}, d\right)=H\left(I_{\text {Shift }_{i j}(G)}, d\right)$.

(iii) Assume there exist vertices $c_{1}, \ldots, c_{k-1}$ such that removing $c_{1}, \ldots, c_{k-1}$ separates $\operatorname{Shift}_{i j}(G)$ into connected components $A_{1}, A_{2}, \ldots, A_{s}$. Let $B=A_{2} \cup \cdots \cup A_{s}$. Since $G$ is $k$-connected, there are vertices $a \in A_{1}$ and $b \in B$ such that $\{a, b\} \in E(G)$ with $\{a, b\} \notin E\left(\operatorname{Shift}_{i j}(G)\right)$. Thus $j=a$ and $a$ is the only vertex in $A_{1}$ which have edges to $B$.

Also, since $\operatorname{Shift}_{i j}(G)$ is an edge shift, if $i \in B$ then $\{i, j\} \in E\left(\operatorname{Shift}_{i j}(G)\right)$. Thus $A$ and $B$ are not separated. Thus we may assume $i \in\left\{c_{1}, \ldots, c_{k-1}\right\}$. Let $i=c=c_{1}$, $\{a, b\} \in E(G)$ and $b \in A_{2}$. We will prove $\{c, p\} \notin E(G)$ for any $p \in A_{2}$.

Assume there exist a vertex $p \in A_{2}$ such that $\{c, p\} \in E(G)$. If $p=b$ then $\{a, b\} \in E\left(\operatorname{Shift}_{c a}(G)\right)$ since $\{a, c\} \in E(G)$. If $p \neq b$ then there is a cycle of $G$ without a chord $\left(\{a, c\},\left\{c, b_{1}\right\}, \ldots,\left\{b_{r}, b\right\},\{b, a\}\right)$ since induced subgraph of $\mathrm{G}$ on $A_{2}$ is connected. But all cycle without a chord is a triangle, since $G$ is chordal. Thus $r=1$ and $b_{1}=b$. Hence $\{a, b\} \in E\left(\operatorname{Shift}_{c a}(G)\right)$.

Thus for any $p \in A_{2}$, we have $\{c, p\} \notin G$. Also $a$ is the only vertex in $A_{1}$ which have edges to $A_{2}$. But removing $a, c_{2}, \ldots, c_{k-1}$ separates $G$ into $A_{1} \backslash\{a\} \cup\{c\}$ and $A_{2}$. This is a contradiction.

Lemma 4.7. Let $G$ be a graph and $\operatorname{Shift}_{i j}(G)$ a disjoint shift.

(i) If $G$ is chordal, then $\operatorname{Shift}_{i j}(G)$ is also chordal.

(ii) $H\left(\tilde{I}_{G}, d\right)=H\left(\tilde{I}_{\text {Shift }_{i j}(G)}, d\right)$.

Proof. If $\operatorname{Shift}_{i j}(G)$ is a disjoint shift, then $C_{i j}(\{j, k\})=\{i, k\}$ for all $\{j, k\} \in G$. This holds chordalness and numbers of complete graphs which are contained in $G$. Thus by the same argument of Lemma 4.6, we have a result. 
Theorem 4.8. Let $G$ be a chordal graph. Then there is $\Delta^{c}(G)$ such that $\Delta^{c}(G)=$ $\Delta^{e}(G)=\Delta^{s}(G)$.

Proof. We already proved $\Delta^{e}(G)=\Delta^{s}(G)$ in $\S 3$. We will construct $\Delta^{c}(G)$ with $\Delta^{c}(G)=\Delta^{e}(G)$ algorithmically.

[Algorithm] Let $G:=G, \Delta:=\emptyset$ and $V:=[n]$.

(I) If $u=\min V$ is a central vertex, then $G:=G \backslash\{u\}, V:=V \backslash\{u\}$ and $\Delta:=\Delta \cup\{\{u, u+1\}, \ldots,\{u, u+\operatorname{deg}(v)\}\}$.

(II) If $u$ is not a central vertex and $G$ has more then 2 connected components which are not isolated vertices, then do $G:=\operatorname{Shift}_{u j}(G)$, where $\operatorname{Shift}_{u j}(G)$ is a disjoint shift.

(III) If $u$ is not a central vertex and $G$ has one connected component which is not an isolated vertex, then for all $v \in V$ with $\{u, v\} \in E(G)$ do $G:=\operatorname{Shift}_{u v}(G)$.

(IV) If $G=\emptyset$ RETERN $\Delta$.

(II) reduce a connected components and (III) and Lemma 4.2 guarantee that we can make a central vertex by edge shifts. Thus this algorithm ends.

Also, if $v$ is a central vertex of chordal graph, then $G \backslash\{v\}$ is also chordal and $K_{\sigma} \subset G \backslash\{v\}$ if and only if $K_{\sigma \cup\{v\}} \subset G$. In the process of the algorithm, this means removing a central vertex does not affect the chordalness and numbers of complete graphs that are contained in $G \cup \Delta$, therefore, holds a Hilbert function of $I_{G \cup \Delta}$. Thus Lemma 4.7 and Lemma 4.6 guarantee this algorithm hold chordalness and Hilbert function of $I_{G \cup \Delta}$. Let $\Delta$ denote a shifted graph which is given by this algorithm. Then $\tilde{I}_{G}$ and $\tilde{I}_{\Delta}$ have a linear resolution and have the same Hilbert function. If $I$ has a linear resolution, then graded Betti numbers of $I$ are determined by its Hilbert function. Thus $\tilde{I}_{G}$ and $\tilde{I}_{\Delta}$ have same graded Betti numbers. By Lemmas 3.2 and 3.3 we have $\Delta=\Delta^{e}(G)$.

J. Herzog conjectured that $\beta_{i j}\left(I_{\Delta^{c}(\sigma)}\right) \geq \beta_{i j}\left(I_{\Delta^{e}(\sigma)}\right) \geq \beta_{i j}\left(I_{\Delta^{s}(\sigma)}\right) \geq \beta_{i j}\left(I_{\sigma}\right)$ for any simplicial complex $\sigma$ and for any positive integers $i$ and $j$. The third inequality was proved by A. Aramova, J Herzog and T. Hibi (see [8, Theorem 8.22]). The first inequality was proved by T. Hibi and me [11, Theorem 3.3]. But the second inequality is still open problem. We don't even know $\beta_{i j}\left(I_{\Delta^{c}(\sigma)}\right) \geq \beta_{i j}\left(I_{\sigma}\right)$.

Recall an argument in [11]. We first proved $m_{\leq k}\left(\Delta^{c}(\sigma)\right) \leq m_{\leq k}\left(\Delta^{e}(\sigma)\right)$ for all $k$. This inequality immediately proves $\beta_{i j}\left(I_{\Delta^{c}(\sigma)}\right) \geq \beta_{i j}\left(I_{\Delta^{e}(\sigma)}\right)$ (see $\left.[11, \S 3]\right)$.

We consider the graph case. For a planar graph, we have the Herzog's conjecture.

Corollary 4.9. Let $G$ be a planar graph. Then one has

(i) $\{4,5\} \notin \Delta^{e}(G)$ and $\{4,5\} \notin \Delta^{s}(G)$.

(ii) $\beta_{i j}\left(I_{\Delta^{s}(G)}\right) \leq \beta_{i j}\left(I_{\Delta^{e}(G)}\right)$.

Proof. $G$ is a subgraph of maximal planar graph. Also, maximal planar graph is chordal and does not contain $K_{5}$. Then by the same argument in Lemma 4.6 (ii), exterior shifting and symmetric shifting of maximal planar graph does not contain $K_{5}$. By the argument above, if $m_{\leq k}\left(\Delta^{e}(G)\right) \leq m_{\leq k}\left(\Delta^{s}(G)\right)$ for any $k$, then we have $\beta_{i j}\left(I_{\Delta^{s}(G)}\right) \leq \beta_{i j}\left(I_{\Delta^{e}(G)}\right)$. Now, Proposition 2.8 and (i) says $m_{\leq k}\left(\Delta^{e}(G)\right) \leq$ 
$m_{\leq k}\left(\Delta^{s}(G)\right)$ for $k=1,2$ and $m_{\leq k}\left(\Delta^{e}(G)\right)=m_{\leq k}\left(\Delta^{s}(G)\right)$ for $k \geq 3$. Thus we have $\beta_{i j}\left(I_{\Delta^{s}(G)}\right) \leq \beta_{i j}\left(I_{\Delta^{e}(G)}\right)$.

Actually, (i) is a known fact. $\{4,5\} \notin \Delta^{s}(G)$ follows from Gluck's work about rigidity. On the other hand, $\{4,5\} \notin \Delta^{e}(G)$ was proved by Kalai. Also, Nevo [10] gives a graph theoretical proof for this fact recently.

Problem 4.10. (i) If $G$ is planar, then $\Delta^{e}(G)=\Delta^{s}(G)$ ?

(ii) Prove $m_{\leq k}\left(\Delta^{e}(G)\right) \leq m_{\leq k}\left(\Delta^{s}(G)\right)$ for any graph $G$ and for any $k$.

Finally, we have following consequence about connectivity of chordal graph.

Corollary 4.11. Let $G$ be a chordal graph. Then the followings are equivalent.

(i) $G$ is $k$-connected;

(ii) $G$ is k-hyperconnected;

(iii) $G$ is generically k-rigid.

Proof. (ii) $\Leftrightarrow$ (iii) is obvious since $\Delta^{e}(G)=\Delta^{s}(G)$. (ii) $\Rightarrow$ (i) is [6, Corollary5.3].

We will prove (i) $\Rightarrow$ (ii). If $G$ is $k$-connected, then we can make $k$ central vertices by algorithm (I) and (III). Also (III) holds connectivity by Lemma 4.6. Thus we have $\{k, n\} \in \Delta^{c}(G)=\Delta^{e}(G)$ for some $\Delta^{c}(G)$.

\section{REFERENCES}

[1] A. Avramova, J. Herzog and T. Hibi, Gotzmann Theorems for exterior algebra and combinatorics, J. Algebra 191, (1997) 174-211.

[2] A. Avramova, J. Herzog and T. Hibi, Ideals with stable Betti numbers, Adv. Math., 152 (2000), no. $1,72-77$.

[3] W. Bruns and J. Herzog, "Cohen-Macaulay rings," Revised Edition, Cambridge University Press, Cambridge, 1996.

[4] P. Erdös, C. Ko and R. Rado, Intersection theorems for systems of finite sets, Quart. J. Math. Oxford Ser. (2) 12 (1961), 313 - 320.

[5] R. Fröberg, On Stanley-Reisner ring, in "Topics in algebra", Banach Center Publications, 26 Part 2, (1990), 57-70.

[6] G. Kalai Hyperconnectivity of graphs, Graphs combin., 1, (1985), 65-79.

[7] G. Kalai, Algebraic shifting, in "Computational Commutative Algebra and Combinatorics" (T. Hibi, Ed.), Advanced Studies in Pure Math., Volume 33, 2002, pp. 121 - 163.

[8] J. Herzog, Generic initial ideals and graded Betti numbers, in "Computational Commutative Algebra and Combinatorics" (T. Hibi, Ed.), Advanced Studies in Pure Math., Volume 33, 2002, pp. $75-120$.

[9] J. Herzog and T. Hibi, componentwise linear ideals, Nagoya Math. J., 153, (1999), 141-153.

[10] E. Nevo, Embeddability and Stresses of Graphs, 2004, arXiv:math.CO/0411009 (preprint).

[11] S. Murai and T. Hibi, The behavior of graded Betti numbers via algebraic shifting and combinatorial shifting, 2005, arXiv:math.AC/0503685 (preprint).

Satoshi Murai

Department of Pure and Applied Mathematics

Graduate School of Information Science and Technology

Osaka University

Toyonaka, Osaka 560-0043, Japan

E-mail:s-murai@ist.osaka-u.ac.jp 